\title{
Non Iterative Formulae for Direct Geodetic Problem
}

\author{
Saad Z. Bolbol ${ }^{1}$, Khaled M. Zaky ${ }^{2}$, Ashraf A. Ghonem ${ }^{3}$ \\ ${ }^{1}$ Professor of Surveying and Geodesy, Shoubra Faculty of Engineering, Benha University, Cairo, Egypt \\ ${ }^{2}$ Associate Professor of Surveying, Shoubra Faculty of Engineering, Benha University, Cairo, Egypt \\ ${ }^{3}$ Lecturer of Surveying, Shoubra Faculty of Engineering, Benha University, Cairo, Egypt
}

Correspondence Author:

Ashraf A. Ghoneim

Lecturer of Surveying, Shoubra Faculty of Engineering, Benha University, Cairo, Egypt

e-mail:dr.ghonem@hotmail.com

Received date: 12 June 2018, Accepted date: 14 September 2018, Online date: 25 September 2018

Copyright: $\odot 2018$ Ashraf A. Ghoneim. This is an open-access article distributed under the terms of the Creative Commons Attribution License, which permits unrestricted use, distribution, and reproduction in any medium, provided the original author and source are credited.

\begin{abstract}
Direct and inverse problem for geodesic on a reference ellipsoid is commonly used for many geodetic applications. Direct problem formulas are used in order to find the geodetic coordinates of the second point of the geodesic line and backward azimuth if geodetic coordinates of the first point, the length of geodesic and forward azimuth are given. It has been common to use iteration in order to calculate such coordinates, however, in this paper; we introduce applicable formulas that do not need iteration in order to be calculated with moderate accuracy. The research formulas results have been compared with the corresponding results of Vincenty's iterative formulas. The comparison showing agreements to within $0.0000004 \mathrm{~km}$ of geodesic length (or $4.0 \mathrm{~mm}$ ) and 0,0001" of azimuth in the range of $4560 \mathrm{~km}$.
\end{abstract}

Keywords: Direct formulas, Geodesic, Geodetic latitude, Inverse formulas, Reduced latitude.

\section{INTRODUCTION}

The direct and inverse problems in geodesy are considered an important task for large number of geodesists. These problems concerns with geodesic lines and azimuths on the ellipsoid. Sjöberg at 2006, 2006a, 2006b and 2007 discussed many solutions for such problems for a geodesic via a power series of the ellipsoidal eccentricity. Although, another solution has been introduced by Heck 1987, Klotz 1991, Schmidt1999 and Schmidt 2006, there is no new due to that they still use series of the ellipsoidal eccentricity. An improved technique is discussed by Thomas and Featherstone at 2005 but the results were agreed with Vincenty's formulas 1975 in the range of geodesic length of $18000 \mathrm{~km}$.

In this research, from a given geodesic length, forward azimuth and geodetic coordinates of the first point, the direct solution gives geodetic coordinates of the second point and the backward azimuth without iteration.

\section{FORMULAE}

If $S$ is the geodesic distance, $\alpha_{12}$ is the forward azimuth, $\varphi_{1}, \varphi_{2}$ are the geodetic latitudes, $\beta_{1}, \beta_{2}$ are the reduced latitudes, $\alpha_{21}$ is the backward azimuth, $\lambda_{1}, \lambda_{2}$ are the longitudes of the two ends of line and $\Delta \theta$ is the central angle of geodesic distance at the centre of ellipsoid. The reference ellipsoid parameters $a, b$ and $e$ are the semi-major, semi-minor axes and eccentricity respectively. The formulae depend on the use of the equivalent spheroid of the reference ellipsoid. Figure (1) shows the geodesic line on ellipsoid and equivalent spheroid.

The reduced latitude $\beta$ is the angle at the centre of a spheroid tangent to the reference ellipsoid along the equator and the radius to the point intersected to the sphere by a straight line perpendicular to the plane of the equator. 
The reduced latitude $(\beta)$ given by:

$$
\beta=\tan ^{-1}(\mathrm{~b} / \mathrm{a}) \tan \varphi
$$

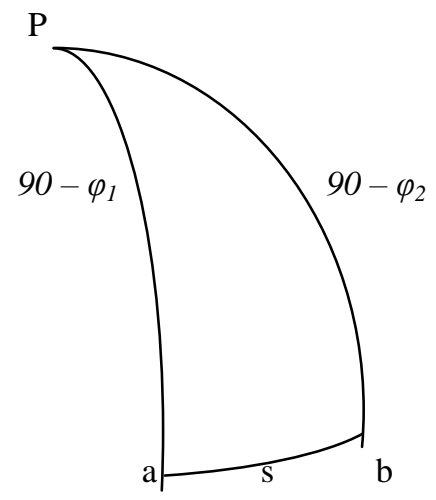

(i) Ellipsoid

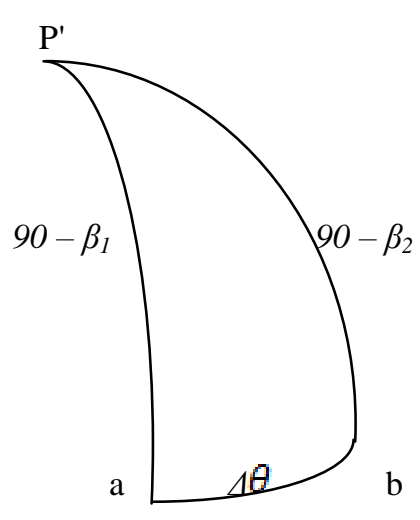

(ii) Spheroid

Figure (1): Ellipsoid and equivalent spheroid

Parametric latitude $\left(\beta_{0}\right)$ which is the latitude perpendicular on the geodesic can be calculated as follows:

$$
\begin{aligned}
& \cos \beta_{0}=\cos \beta_{1} \sin \alpha_{12}=-\cos \beta_{2} \sin \alpha_{21} \\
& \sin \theta_{1}=\sin \beta_{1} / \sin \beta_{0} \\
& \Delta \theta^{*}=\alpha(\mathrm{S} / \mathrm{b})-\beta \sin \Delta \theta \cos 2 \sigma+\gamma \sin 2 \Delta \theta \cos 4 \sigma-\gamma \sin 3 \Delta \theta \cos 6 \sigma+ \\
& \quad \delta \sin 4 \Delta \theta \cos 8 \sigma
\end{aligned}
$$

Where

$$
\begin{aligned}
& 2 \sigma=2 \Delta \theta_{1}+\Delta \theta \\
& \sin \Delta \theta_{1}=\sin \beta_{1} /\left\{\cos ^{-1}\left(\cos \beta_{1} \sin \alpha_{12}\right)\right\} \\
& \mathrm{S}^{\prime}=b\left(A_{0} \Delta \theta^{r}+B_{0} \sin \Delta \theta \cos 2 \sigma-C_{0} \sin 2 \Delta \theta \cos 4 \sigma+D_{0} \sin 3 \Delta \theta \cos 6 \sigma\right) \\
& d \Delta \theta=\alpha\left(\frac{s-s^{\circ}}{b}\right)
\end{aligned}
$$

The geodetic latitude $\varphi_{2}$ is calculated as:

$$
\sin \beta_{2}=\cos \beta_{1} \sin \Delta \theta \cos \alpha_{12}
$$

And

$$
\tan \varphi_{2}=\frac{a}{b} \tan \beta_{2}
$$

The back azimuth is calculated from the following equation:

$$
\sin \alpha_{21}=\left(\cos \beta_{1} \sin \alpha_{12}\right) / \cos \beta_{2}
$$

The difference of longitude is obtained as follows:

$$
\begin{aligned}
& \Delta \lambda=\cos ^{-1}\left(-\cos \alpha_{12} \cos \alpha_{21}+\sin \alpha_{12} \sin \alpha_{21} \cos \Delta \theta\right)+\cos \beta_{1}(A \Delta \theta \\
& +B \sin \Delta \theta \cos 2 \sigma-C \sin 2 \Delta \theta \cos 4 \sigma+D \sin 3 \Delta \theta \cos 6 \sigma) \\
& \lambda_{2}=\lambda_{1}+\Delta \lambda
\end{aligned}
$$


Where

$$
\begin{aligned}
& A_{0}=1+\frac{k^{2}}{4}-\frac{a k^{4}}{64}+\frac{5 k^{6}}{256}-\frac{175 k^{8}}{16384}, \\
& B_{0}=\frac{k^{2}}{4}-\frac{k^{4}}{16}+\frac{15 k^{6}}{256}-\frac{25 k^{8}}{2045}, \\
& C_{0}=\frac{k^{4}}{128}+\frac{3 k^{6}}{512}-\frac{35 k^{8}}{8192}, \\
& D_{0}=\frac{3 k^{6}}{1536}-\frac{5 k^{8}}{6144} \text {, } \\
& k \text { is the modulus and equal to } e \sin \beta_{0} \\
& \propto=\frac{1}{A_{0}} \rho^{a}, \\
& \mathrm{~B}=\frac{\mathrm{B}_{0}}{\mathrm{~A}_{0}} \rho{ }^{\sigma} \text {, } \\
& \gamma=\frac{C_{0}}{A_{0}} \rho^{\mathrm{m}}, \\
& \delta=\frac{D_{0}}{A_{0}} \rho^{m}, \\
& \rho^{\mathrm{I}}=206265 \text {. } \\
& A=e^{2}\left(\frac{1}{2}+\frac{e^{2}}{8}+\frac{e^{4}}{16}+\frac{5 e^{6}}{128}+\frac{7 e^{8}}{256}\right)-\frac{\left(e^{4} \sin ^{2} \beta_{0}\right)}{16}+\left(1+e^{2}+\frac{15 e^{2}}{16}\right)+\frac{a}{128} e^{2} \sin ^{4} \beta_{0}\left(1+\frac{15 e^{2}}{8}\right)-\frac{25 e^{6}}{2048} \sin ^{6} \beta_{0} . \\
& \mathrm{B}=\frac{e^{4}}{16} \sin ^{2} \beta_{0}\left(1+\mathrm{e}^{2}+\frac{15 \mathrm{e}^{4}}{16}\right)-\frac{\mathrm{e}^{6}}{32} \sin ^{4} \beta_{0}\left(1+\frac{15 \mathrm{e}^{2}}{8}\right)+\frac{75 \mathrm{e}^{8}}{4096} \sin ^{6} \beta_{0}, \\
& C=\frac{\theta^{6}}{256} \sin ^{4} \beta_{0}\left(1+\frac{15}{8} e^{2}\right)-\frac{15}{4096} e^{8} \sin ^{6} \beta_{0} \text { and } \\
& \mathrm{D}=\frac{5}{1228 \mathrm{~g}} \mathrm{e}^{\mathrm{g}} \sin ^{6} \beta_{0}
\end{aligned}
$$

For more accurate results parameters can be expanded.

\section{NUMERICAL TEST}

For checking the accuracy of the proposed formulas and its consistency check with other method tested for solving direct geodetic problem before, we performed a test. Table (1) contains ten GPS stations of a geodetic network selected for the required test. Vincenty's formulas are also selected for comparing its results with our formulas results to judge on the accuracy of our formulas. The differences between the proposed formulas results and those from Vincenty's formulas are listed in Table (2).

Table (1): Selected Points with their Geodetic Coordinates

\begin{tabular}{|c|c|c|c|c|c|c|}
\hline \multirow{2}{*}{ Station } & \multicolumn{3}{|c|}{$\boldsymbol{\Phi}$} & \multicolumn{2}{c|}{$\boldsymbol{\lambda}$} \\
\cline { 2 - 7 } & ${ }^{\circ}$ & $'$ & " & ${ }^{\circ}$ & $\cdot$ & " \\
\hline ZOO & 22 & 08 & 41.12054 & 36 & 43 & 13.85822 \\
\hline Z04 & 24 & 26 & 15.03729 & 27 & 28 & 31.49535 \\
\hline Z09 & 26 & 01 & 02.76559 & 34 & 19 & 16.33949 \\
\hline Z10 & 25 & 57 & 20.06853 & 32 & 09 & 24.25427 \\
\hline Z15 & 29 & 21 & 00.09509 & 34 & 46 & 20.60622 \\
\hline Z16 & 29 & 21 & 00.09509 & 34 & 46 & 20.60622 \\
\hline Z19 & 29 & 50 & 02.97004 & 30 & 36 & 04.07107 \\
\hline Z22 & 31 & 26 & 16.24367 & 25 & 23 & 55.08191 \\
\hline Z24 & 29 & 29 & 39.45264 & 27 & 10 & 06.52319 \\
\hline Z29 & 22 & 02 & 39.31849 & 25 & 16 & 54.39147 \\
\hline
\end{tabular}


Citation: Saad Z. Bolbol et al., Non Iterative Formulae for Direct Geodetic Problem. Australian Journal of Basic and Applied Sciences, 12(9): 131-134 D0I: 10.22587/ajbas.2018.12.9.22

Table (2): Differences between Our Results and the Ones from Vincenty's Solution for the Direct Geodetic Problem (from the Origin of ZOO)

\begin{tabular}{|c|c|c|c|c|c|c|}
\hline \multirow{2}{*}{ Station } & $\mathrm{S}(\mathrm{km})$ & \multicolumn{3}{|c|}{$\alpha$} & \multirow{2}{*}{$\Delta \mathrm{s}(\mathrm{km})$} & $\Delta \alpha\left({ }^{\prime \prime}\right)$ \\
\cline { 3 - 6 } & & $\circ$ & $\prime$ & $"$ & & \\
\hline Z04 & 296.8308373 & 350 & 15 & 19.7654 & 0.0000001 & 0.0000 \\
\hline Z09 & 4560.7395641 & 342 & 33 & 23.8765 & 0.0000003 & 0.0000 \\
\hline Z10 & 421.1812933 & 338 & 34 & 31.4178 & 0.0000002 & 0.0001 \\
\hline Z15 & 255.8765365 & 325 & 54 & 54.5972 & 0.0000004 & 0.0001 \\
\hline Z16 & 165.8743212 & 334 & 32 & 45.9125 & 0.0000002 & 0.0001 \\
\hline Z19 & 432.4532167 & 330 & 43 & 56.7531 & 0.0000000 & 0.0000 \\
\hline Z22 & 190.000 .0000 & 315 & 23 & 45.7686 & 0.0000000 & 0.0000 \\
\hline Z24 & 342.6742198 & 321 & 52 & 48.3652 & 0.0000001 & 0.0000 \\
\hline Z29 & 234.6754329 & 265 & 24 & 35.3683 & 0.0000002 & 0.0001 \\
\hline
\end{tabular}

\section{CONCLUSION}

The above proposed formulas of this research solved the direct problem without iterations. These formulas calculations relate to the reference ellipsoid with the equivalent spheroid. For many purposes, it is needed to calculate the geodetic coordinates of terminal points of geodesic and these formulas are easy to use avoiding iterations. The tests show that the results of our direct problem solution coincide with Vincenty's method. The proposed method of this research is an independent method for checking Vincenty's method and vice versa. Differences between the results of the research method and those obtained from Vincenty's formulas for the direct geodetic problem did not exceed $0.0000004 \mathrm{~km}(4 \mathrm{~mm})$ in geodesic length and $0.0001 "$ in azimuth in range of $4960 \mathrm{~km}$. These differences may be considered much smaller.

\section{REFERENCES}

1. Bolbol, S. (2018). "Surveying and Geodetic Academic Applications", Lambert Germany Publications, 2018.

2. Bolbol, S.(1978)."Accurate inverse formulae for geodesic calculation", Department of geodesy, The University of Oxford.

3. Heck, B. (1987). Rechenverfahren und Auswertemodelle der Landesv- ermessung, Herbert Wichmann Verlag, Karlsruhe, Germany.

4. Kamey,C . F. F.(2013), “Algorithms for geodesics", Link http://geom-raphiclib.sourceforge.net/geod.html E-print arXiv: 1109.4448.

5. Klotz, J. (1991). "Eine analytische Lösung kanonischer Gleichungen der geodätischen Linien zur Transformation ellipsoidischer Flächenkoor-dinaten.”Deutsche Geod. Kommission Ser. C, No. 385, BavarianAcademy of Sciences, Munich.

6. Lars E. Sjöberg and Masoud Shirazian (2012). "Solving the Direct and Inverse Geodetic Problems on the Ellipsoid by Numerical Integration", Journal of Surveying Engineering, Vol. 138, No. 1, DOI: 10.1061/(ASCE)SU.1943-5428.0000061. @ 2012 American Society of Civil Engineers.

7. Schmidt, H. (2006). "Note on Lars E. Sjöberg: New solutions to the direct and indirect geodetic problems on the ellipsoid." Z. Vermessungsw., 131, 153-154.

8. Schmidt, H. (1999). "Lösung der geodätischen Hauptaufgaben auf dem Rotationsellipsoid mittels numerischer Integration." Z. Vermessungsw. 124, 121-128.

9. Sjöberg, L. E. (2006a). "New solutions to the direct and indirect geodetic problems on the ellipsoid." Zeitschrift fuer Vermessungswesen, 131, 35-39.

10. Sjöberg, L. E. (2006b). “Comment to H. Schmidt's remarks on Sjöberg, zfv 1/2006, 35-39.” Z. Vermessungsw., $131,155$.

11. Sjöberg, L. E. (2006c). "Determination of areas on the plane, sphere and ellipsoid." Surv. Rev., 38, 583-593. Sjöberg, L. E. (2007). "Precise determination of the Clairaut constant inn ellipsoidal geodesy." Surv. Rev., 39(303), 81-86.

12. Thomas, C. M., and Featherstone, W. E. (2005). "Validation of Vincenty's formulas for the geodesic using a new fourth-order extension of Kivioja's formula." J. Surv. Eng., 131(1), 20-26.

13. Vincenty, T. (1975) "Direct and inverse solutions of geodesics on ellipsoid with application of nested equations" Survey review 23 (175): 88-93. 